\title{
Percepción y actitud de los estudiantes universitarios respecto al cambio climático
}

\author{
Perception and attitude of university students \\ regarding climate change
}

\author{
Miranda Velázquez Elizabeth \\ Universidad Técnica de Machala \\ emiranda@utmachala.edu.ec \\ Betancourt Quintero Bárbara \\ Universidad Técnica de Machala \\ bbetancourt@utmachala.edu.ec \\ Reyes Romero José \\ Universidad Técnica de Machala \\ jmreyes@utmachala.edu.ec
}

\section{Velásquez Morocho Brenda \\ Universidad Técnica de Machala bvelasque1@utmachala.edu.ec}

Santos Luna Jovanny Universidad Técnica de Machala jsantos@utmachala.edu.ec

Gallegos Gallegos Edith Universidad Técnica de Machala egallegos@utmachala.edu.ec

\section{Revista Cumbres Vol.7 №1}

Versión electrónica ISSN 1390-3365 http://investigacion.utmachala.edu.ec/revistas/index.php/Cumbres http://doi.org/10.48190/cumbres.v7n1a3 


\title{
RESUMEN
}

El cambio climático constituye un peligro para la salud humana y ambiental global. El personal de salud debe ejercer funciones de protección, promoción, vigilancia y prevención, teniendo en cuenta las competencias, habilidades y actitudes que adquirieron para incorporarse como profesionales en el campo de la salud, por lo tanto, debe fomentar ecoestrategias comunitarias en educación ambiental para su preservación. El presente estudio identificó la percepción y actitud frente al cambio climático de los estudiantes universitarios de Enfermería de la Universidad Técnica de Machala. Investigación científica, transversal, descriptiva y analítica efectuada en la población universitaria de la carrera de Enfermería durante los meses de mayo - septiembre del 2019. El tamaño de la muestra fue de 287 sujetos. Se aplicó un muestreo aleatorio simple, con cuestionario avalado de quince preguntas cerradas en escala Likert. El porcentaje de información, se evidenció así: 32 alumnos plasmaron que el cambio climático surge por causas naturales y humanas lo que corresponde al 11,15\% de la población total estudiada. Además, encontramos que no todos los estudiantes adoptaron medidas individuales para mitigar los riesgos ambientales, pero sí conocen de sus causas y efectos. El personal de salud debe difundir a la comunidad la creación de entornos saludables para prevenir enfermedades y salvar al planeta de la degradación ambiental, además, diseñar un programa en educación ambiental, cuyas acciones estén orientadas a que el individuo tome conciencia de la importancia de modificar sus actitudes frente a las problemáticas ambientales suscitadas en la actualidad.

Palabras clave: Cambio climático, salud ambiental, educación para la salud.

\begin{abstract}
Climate change constitutes a danger to global human and environmental health. Health personnel must exercise protection, promotion, surveillance and prevention functions, taking into account the competencies, skills and attitudes they acquired to join as professionals in the field of health, therefore, they must promote community eco-strategies in environmental education to its preservation. The present study identified the perception and attitude towards climate change of university nursing students of the Technical University of Machala. Scientific, cross-sectional, descriptive and analytical research carried out in the university population of the nursing career during the months of May - September 2019. The sample size was 287 subjects. Simple random sampling was applied, with a guaranteed questionnaire of fifteen closed questions on a Likert scale. The percentage of information was
\end{abstract}


evidenced as follows: 32 students stated that climate change arises from natural and human causes, which corresponds to $11.15 \%$ of the total population studied. Furthermore, we found that not all students adopted individual measures to mitigate environmental risks, but they do know about their causes and effects. Health personnel must disseminate to the community the creation of healthy environments to prevent diseases and save the planet from environmental degradation, in addition, design an environmental education program, whose actions are aimed at making the individual aware of the importance of modifying their attitudes towards environmental problems raised today.

Keywords: Climate change, environmental health, health education.

\section{INTRODUCCIÓN}

El cambio climático constituye un peligro inminente para la salud humana y ambiental global. La degradación ambiental ha pasado a ser un importante factor de riesgo, por el impacto de los seres humanos en la contaminación del aire, los suelos, mares y océanos, con componentes químicos, físicos, y biológicos. Además el cambio climático contribuye a la inseguridad alimentaria, la pobreza, y en general a aumentar la vulnerabilidad de la población (Zamora Martínez, 2015) (Martínez y Peña, 2017).

La Organización Mundial de la Salud (OMS 2015), plasma en diversos estudios la influencia que ejerce el cambio climático sobre los determinantes sociales y medioambientales de salud, al no tener cubierta las necesidades en alimentación, energía, agua, y vivienda; solicita además que a nivel global, cada país preserve la salud contra el cambio climático, pues entre el 2030 y el 2050 esta problemática podría causar 25.000 muertes adicionales (Sánchez Zavaleta, 2016).

Según la Organización de Naciones Unidas (ONU), el cambio climático repercute de manera negativa en la salud de la población por estrés calórico, y sus consecuencias son: malnutrición e infecciones por vectores (paludismo y dengue), cáncer pulmonar, accidentes cerebrovasculares, cardiopatías isquémicas, y otras dolencias sanitarias provocadas por la contaminación atmosférica (Malagón Rojas et al., 2017) (Flores, 2018) (Heras et al., 2017).

En América Latina, especialmente Centroamérica, el efecto invernadero y la falta de seguridad alimentaria, son los principales ejes por los cuales el bienestar y la supervivencia humana se ven afectadas a gran magnitud. A los Gestores de medios comunicativos en radio/Tv, y al personal de salud, les corresponde un importante papel, en concientizar a la comunidad acerca de los riesgos del cambio climático, con la finalidad de menguar la extinción de flora - fauna, y tratar de reducir enfermedades asociadas al calentamiento global; estos aspectos contribuirán a mejorar la calidad de vida a mediano y largo plazo (Hernández y Danae, 2016) (Estrada et al., 2016).

En el Ecuador, las autoridades del ministerio del Ambiente señalan, que el cambio climático ha afectado de manera considerable a ciertos sectores 
de producción: los agricultores, el sector camaronero y pesquero; en la actualidad no se pueden reconocer el inicio o el fin de las temporadas lluviosas y calurosas, y esto ha afectado la productividad en el país, además que los inviernos son más agresivos y las olas de calor más intensas (Toulkeridis et al., 2020).

Las acciones de promoción por parte del personal de salud son fundamentales para modificar las problemáticas ambientales: fomentar la participación en campañas medioambientales, instruir sobre la necesidad del ahorro de energía, la separación de desechos en los contenedores de reciclaje, la reconstrucción de manglares, bosques y aguas continentales, la importancia de reciclar, hervir el agua y ahorrarla, ahorrar electricidad, plantar árboles en las comunidades, la ventilación adecuada de viviendas, fomentar el uso de las bicicletas, andar a pie y el uso de transporte público en las ciudades, etc.

El presente estudio identificó la percepción y actitudes ante el cambio climático de los estudiantes universitarios de la carrera de Enfermería de la Universidad Técnica de Machala (UTMACH), debido a la importancia que tiene una adecuada preparación del personal sanitario en la promoción y prevención de salud, incluida la salud ambiental y ecológica.

\section{MATERIALES Y MÉTODOS}

Se realizó un estudio descriptivo de corte transversal, campo, analítico, cuali-cuantitativo, acerca de la percepción y actitud ante el cambio climático de los universitarios de Enfermería de la UTMACH. El universo estuvo constituido por los 673 estudiantes de la carrera de Enfermería de los cuales fueron encuestados 287 estudiantes, en los meses de mayo - septiembre del 2019. Se aplicó un muestreo aleatorio simple con la técnica del cuestionario. Se utilizó La encuesta sobre "la percepción de la ciudadanía Viguesa sobre el cambio climático", elaborado por el equipo técnico de la Oficina do Valedor do Cidadán, modificando el cuestionario aplicado por la Fundación Mapfre en varios informes, el cual consta de 15 preguntas cerradas en escala Likert y 98 ítems (Espada Recarey et al., 2016). Todos los estudiantes dieron el consentimiento informado a la investigación. Se utilizó el programa estadístico IBM SPSS Statistics Versión 22 para analizar y fundamentar la información que se obtuvo en la investigación.

\section{RESULTADOS Y DISCUSIÓN}

Mediante el análisis descriptivo y de campo, con relación a la distribución de estudiantes de Enfermería UTMACH encuestados por edades y sexo, pudimos conocer que la muestra estuvo conformada por 189 mujeres y 98 hombres, cuya edad oscilan entre 20-24 años, con una media de 22,3 años como corresponde con las edades de la etapa de ciclo de estudio universitario (Tabla $\mathrm{N}^{\circ} 1$ ). 
Tabla $\mathrm{N}^{\circ} 1$. Distribución de edad universitaria

\begin{tabular}{|l|l|l|l|l|}
\hline \multicolumn{6}{|c|}{ DISTRIBUCIÓN POR RANGO DE EDADES } \\
\hline \multirow{6}{*}{ Rango de Edades } & N (\%) & Masculino & Femenino \\
\hline \multirow{3}{*}{ VALIDO } & $18-19$ & $66(23,0)$ & 25 & 41 \\
\cline { 2 - 5 } & $20-24$ & $167(58,2)$ & 56 & 111 \\
\hline & $25-29$ & $43(15,0)$ & 16 & 27 \\
\cline { 2 - 5 } & $30-34$ & $11(3,8)$ & 1 & 10 \\
\hline
\end{tabular}

Fuente: Programa Estadístico IBM SPSS Statistics Versión 22

La Clasificación de distribución por edad y sexo muestra que el rango oscilatorio entre 20 - 24 años incluye la mayor frecuencia con 167, seguido de 18 - 19 años con 66 estudiantes. Se destaca además el sexo femenino con 189 alumnas, para 98 del género masculino, lo que corresponde con las edades de inicio del sistema educativo universitario (porcentaje de población equivalente al 58,2\%), y la característica habitual de la carrera que es practicada más por mujeres (Suárez Zozaya, 2017).

En las primeras preguntas se indagó acerca de la percepción que poseen los estudiantes sobre las causas del cambio climático (Tabla $\mathrm{N}^{\circ} 2$ ). El porcentaje de información se evidenció de la siguiente manera: el 11,2\%, indicó que es exclusivamente por causas naturales; a su vez un 4,9\% atribuye principalmente los causales naturales como responsable de los fenómenos climáticos (variaciones en la órbita de la Tierra, variabilidad solar, movimiento tectónico de placas y actividad volcánica); 195 estudiantes contestan que el fenómeno climático se debe principalmente a causas humanas (deforestación e incendios vegetales) lo que equivale al 67,9\%; 14 educandos describieron que el calentamiento planetario surge exclusivamente por la actividad humana (quema de combustibles fósiles, abuso de vehículos convencionales), representando un 4,9\%, así mismo, 32 alumnos plasmaron en el cuestionario que hoy en día el cambio climático surge por causas naturales y humanas lo que corresponde al 11,1 \% de la población total estudiada.

Tabla $N^{\circ} 2$. Causas de los Fenómenos climáticos

\begin{tabular}{|c|c|c|c|}
\hline \multicolumn{4}{|c|}{ FENÓMENO CLIMÁTICO } \\
\hline & & $\mathbf{n}$ & $\%$ \\
\hline \multirow[t]{5}{*}{ VÁLIDO } & $\begin{array}{l}\text { Exclusivamente por } \\
\text { causas naturales }\end{array}$ & 32 & 11,2 \\
\hline & $\begin{array}{l}\text { Principalmente por } \\
\text { causas naturales }\end{array}$ & 14 & 4,9 \\
\hline & $\begin{array}{l}\text { Principalmente por } \\
\text { causas humanas }\end{array}$ & 195 & 67,9 \\
\hline & $\begin{array}{l}\text { Exclusivamente por } \\
\text { causas humanas }\end{array}$ & 14 & 4,9 \\
\hline & $\begin{array}{l}\text { Causas naturales y } \\
\text { humanas }\end{array}$ & 32 & 11,1 \\
\hline Total & 287 & 287 & 100,0 \\
\hline
\end{tabular}

Fuente: Programa Estadístico IBM SPSS Statistics Versión 22 
Carlos Sánchez en el año 2016, publicó un estudio sobre la evolución del concepto de cambio climático y su impacto en la salud pública del Perú, donde se indicó que hubo un pánico inicial, ya que en este país se ha dado problemas complejos relacionados a la deforestación, degradación de bosques, falta de agua potable en múltiples comunidades, derrames de petróleo, explotación minera; todos ellos factores humanos directamente causales del cambio climático. La emisión de gases por el efecto invernadero, la variabilidad solar y el movimiento tectónico de placas han afectado de manera drástica los suelos forestales y son las causas principales por las cuales el cambio climático ha estado deteriorado en los últimos años (Sánchez Zavaleta, 2016). Si se realiza una correlación con el presente estudio de investigación, consideramos que las causas humanas son las más percibidas como responsables del cambio climático.

Una posible estrategia para mitigar estos efectos adversos climáticos, es la construcción de políticas saludables, tales como: reconstrucción de manglares, bosques y aguas continentales, reciclar, hervir agua necesaria, ahorrar electricidad, plantar árboles, ventilación adecuada de viviendas, fomentar el uso de las bicicletas y transporte público en la ciudad, etc. (Kaeslin et al., 2015).

Durante el análisis e interpretación de los datos obtenidos en relación a la percepción de la afectación de la salud como consecuencias del cambio climático, se encontró que, de los estudiantes encuestados en la investigación 115 (40,1\%), contestaron que hay una alta probabilidad de que el cambio climático afecte a la salud poblacional, mientras que el 32,4\% consideran bastante probable la afección de la salud humana por la degradación ambiental; poco probable con un 18,8\% y nada probable un 7,3\%. Por otra parte, 4 alumnos desconocían sobre el impacto en salud del cambio climático, representando el 1,4\% (Tabla $\mathrm{N}^{\circ} 3$ ).

Tabla $N^{\circ} 3$. Afectación de la salud por el cambio climático

\begin{tabular}{|l|l|l|l|}
\hline \multicolumn{5}{|c|}{ AFECCIÓN EN LA SALUD HUMANA } \\
\hline \multirow{3}{*}{ VÁLIDO } & $\mathbf{n}$ & $\%$ \\
\hline & Nada probable & 21 & 7,3 \\
\cline { 2 - 5 } & Poco probable & 54 & 18,8 \\
\cline { 2 - 5 } & Bastante probable & 93 & 32,4 \\
\cline { 2 - 5 } & Muy probable & 115 & 40,1 \\
\cline { 2 - 5 } & Desconozco & 4 & 1,4 \\
\hline Total & 287 & 100,0 \\
\hline
\end{tabular}

Fuente: programa estadístico IBM SPSS statistics versión 22

Farid Rodríguez, María Jiménez, y Lilibeth Pedraza en el año 2019 mencionaron en su investigación científica, que el fenómeno ambiental se puede considerar como un escenario epidemiológico producto del impacto causado por los patrones del clima, el cual ha incidido en la actividad económica y en los ecosistemas. Estos efectos a largo plazo influirán sobre la salud huma-

\section{Cumbres}


na ocasionando enfermedades tropicales transmitidas por vectores, asma, tuberculosis, parasitosis, infarto agudo de miocardio, tracoma, depresión, psicopatías mentales, etc. Consecuentemente, es esperable la ampliación de patologías para los años comprendidos entre 2030 - 2050 producto del daño climático (Rodríguez et al., 2019). En base a la investigación efectuada y previa charla educativa a los futuros profesionales de la salud del área de Enfermería, se instó a que cada uno de ellos se plantee alianzas organizacionales en pro beneficio de la salud para empoderar y mejorar el sistema de salud. El pilar fundamental de la promoción en pro de la salud ambiental lo constituyen los medios de comunicación y el personal de salud.

Los medios informativos (televisión, radio), son actores claves en el sistema de salud, dado que la población tiende a escucharlos y seguirlos activamente; por lo cual, realizar actividades, propagandas, programas al respecto es ideal para la adopción de comportamientos sanos sobre el cuidado del medio ambiente. Por ello, también es factible que el profesional sanitario difunda y aplique la participación activa, individual y comunitaria, para fomentar el cuidado ambiental y el autocuidado en salud (Busse y Godoy, 2016) (Rodriguez et al., 2018) (Rodríguez Cruz y Bezunartea Valencia, 2016).

En base a las preguntas de encuesta realizada sobre si alguna vez ha tomado personalmente alguna medida para aminorar la degradación ambiental en los últimos años, los resultados arrojan que el 59.2\% refirió que nunca ha tomado medidas para evitar consecuencias de los efectos climáticos, en cambio un $33.8 \%$ responde que de vez en cuando lo realizan y el $7 \%$ casi siempre.

Tabla $\mathrm{N}^{\circ}$. Medidas para mitigar el cambio climático en los últimos años

\begin{tabular}{|l|l|l|l|}
\hline \multicolumn{5}{|c|}{ MEDIDAS PARA MITIGAR EL CAMBIO CLIMÁTICO } \\
\hline \multirow{2}{*}{ VÁLIDO } & n & $\%$ \\
\hline & Nunca & 170 & 59,2 \\
\cline { 2 - 5 } & Casi siempre & 20 & 7,0 \\
\cline { 2 - 5 } & De vez en cuando & 97 & 33,8 \\
\hline Total & & 287 & 100,0 \\
\hline
\end{tabular}

Fuente: Programa Estadístico IBM SPSS Statistics Versión 22

Muchos estudios dan fe que, si bien las personas conocen el cambio climático no se animan a un cambio de actitud individual en el seno de su hogar y comunidad. Si bien los estudiantes reciben información acerca del tema, el 70\% no ha conversado en su hogar sobre la problemática, el $73 \%$ señala que en su institución educativa no han desarrollado contenidos sobre el cambio climático, el $78 \%$ indica que no ha leído en la prensa reportajes o informaciones sobre el cambio climático y el $75 \%$ reporta que no ha escuchado información en los medios de comunicación, lo cual demuestra lo mucho que falta para fomentar una cultura ambiental saludable (González Ordóñez, 2016).

Lo que si queda demostrado es que, existen distintas actitudes de los sujetos frente al cambio climático, unos optan por disimular al decir que ocurre 
como consecuencia de los ambientalistas, algunos procuran subestimarlo porque el hombre cuenta con la capacidad de crear tecnología ambientalista, ciertos individuos optan por agravarlo al deforestar árboles y otros entes suelen ser indolentes, es decir, ocurre un problema con el medio ambiente, y a ellos no les corresponde inventar o dar soluciones a las problemáticas suscitadas con el ecosistema (Ochoa et al., 2015).

Es necesario, pautar metas con la finalidad de mejorar el sistema de salud y que éste vaya acorde a la armonía con el entorno para mejorar la calidad de vida (Moreno, 2015) (Coronel Carbo y Marzo Páez, 2017). El personal de salud debe diseñar programas efectivos de educación ambiental, cuyas acciones estén orientadas a que el individuo tome conciencia de la importancia de modificar sus actitudes frente a las problemáticas ambientales (Jacobi y Maia, 2016).

\section{CONCLUSIONES}

El cambio climático constituye un peligro inminente para la salud humana y ambiental global, el personal de salud debe ejercer funciones de protección, promoción, vigilancia y prevención, teniendo en cuenta las competencias, habilidades y actitudes que adquirieron para incorporarse como profesionales en el campo de la salud, por lo tanto, debe fomentar ecoestrategias comunitarias en educación ambiental para su preservación. La degradación ambiental es el causal del cambio climático, el factor humano incide en su afectación, al contaminar aire, suelos, mares y océanos con componentes químicos, físicos, biológicos, o a su vez la inseguridad alimentaria lo que aumenta la vulnerabilidad de la población.

Si bien muchos estudiantes de Enfermería presentan conocimiento sobre las causas y efectos del cambio climático, así como las medidas a mitigar para reducir de manera significativa los riesgos ambientales, se encontró resultados desfavorables, pues presentan una actitud indolente frente a la degradación ambiental, sin poner en práctica actitudes ambientalistas desde su ser, su hogar y en la comunidad. El personal de Enfermería debe promover entornos saludables, y desde el cuidado establecer acciones de prevención en salud, y salvar al planeta de la degradación ambiental y sus consecuencias. Establecer estrategias psicoeducativas desde la intervención, puede servir de fortaleza para cambios actitudinales en los estudiantes, y la sensibilización de la problemática en el individuo y la comunidad, de manera que se tome conciencia de la importancia de modificar estilos de vida, para vivir en un ambiente ecológicamente saludable y sustentable.

Otras investigaciones deben realizarse, e identificar las causales actitudinales frente al cambio climático, y establecer a partir de ello un abordaje estratégico de actuación.

\section{Cumbres}




\section{REFERENCIAS BIBLIOGRÁFICAS}

Busse, P., y Godoy, S. (2016). Comunicación y salud. Psicología y Salud, 14(38), 1-35. https://scielo.conicyt.cl/scielo.php?script=sci_arttext\&pi$\mathrm{d}=\mathrm{S} 0719-367 \mathrm{X} 2016000100001$

Coronel Carbo, J., y Marzo Páez, N. (2017). La promoción de salud para la creación de entornos saludables en América Latina y el Caribe. Medisan, 21(12), 3415-3423. https://doi.org/ISSN 1029-3019

Espada Recarey, L., Martínez Cacharrón, V. M., Iradiel Sánchez, J., Sobrino Balboa, J. L., Rey González, G., Cabral, G., Comesaña Romero, M., Cuadrado Rodríguez, D., González Rodriguez, I., Pérez Quintero, C. R., y Rodríguez Rodríguez, F. J. (2016). Percepción de la ciudadanía viguesa sobre el cambio climático. http://hoxe.vigo.org/pdf/valedorcidadan/percepcion_cc.pdf

Estrada, D. A., Gallo, D. M., y Nuñez, D. E. (2016). Contaminación ambiental, su influencia en el ser humano, en especial: el sistema reproductor femenino. Revista Universidad y Sociedad, 8(3), 1-6. https://doi.org/ISSN 2218-3620

Flores, R. (2018). El cambio climático en las representaciones sociales de los estudiantes universitarios. Revista Electronica de Investigacion Educativa, 20(1), 122-132. https://doi.org/10.24320/redie.2018.20.1.1443

González Ordóñez, A. (2016). Programa de Educación Ambiental sobre el cambio climático en la educación formal y no formal. Revista Universidad y Sociedad, 8(3), 99-107. http://scielo.sld.cu/scielo.php?script=sci_arttext\&pid=S2218-36202016000300013

Heras, F., Meira, P., y Justel, A. (2017). La percepción social de los riesgos del cambio climático sobre la salud en España. Revista de Salud Ambiental, 17(1), 40-46. https://ojs.diffundit.com/index.php/rsa/article/view/842

Hernández, A., y Danae, L. (2016). Cambio climático y agricultura: una revisión de la literatura con énfasis en América Latina. El Trimestre Económico, LXXXIII (4(332), 459-496. http://www.scielo.org.mx/pdf/ete/ v83n332/2448-718X-ete-83-332-00459.pdf

Jacobi, P. R., y Maia, R. D. E. A. (2016). Desafíos y Estrategias para fortalecer las relaciones entre ciencia y política en relación al Cambio Climático. Ambiente \& Sociedade, XIX(4), 195-210. https://www.scielo.br/scielo. php?pid=S1414-753X2016000400235\&script=sci_arttext\&tlng=es

Kaeslin, E. ;, Redmond, I., y Dudley, N. (2015). Medidas de adaptación al cambio climático. In La fauna silvestre en un clima cambiante (FAO, pp. 6183). Departamento Forestal, FAO. http://www.fao.org/docrep/017/i2498s/ i2498s.pdf

Malagón Rojas, J., Garrot Wilches, C., y Castilla Bello, P. (2017). Cambio climático y salud humana: una revisión desde la perspectiva colombiana. Salud Unnimorte Scielo, 33(2), 224-241. http://www.scielo.org.co/pdf/sun/ 
v33n2/2011-7531-sun-33-02-00224.pdf

Martínez, M., y Peña, E. (2017). Percepción sobre educación ambiental y medio ambiente en estudiantes de 1er año de la carrera de agronomía de la Universidad de las Tunas. Dialnet, 4(48), 5. https://dialnet.unirioja.es/ servlet/articulo?codigo $=6105591$

Moreno, J. (2015). Las actitudes de las personas frente al cambio climático Enviado. In Cambio Climático - org. https://www.cambioclimatico.org/contenido/las-actitudes-de-las-personas-frente-al-cambio-climatico

Ochoa, M., Castellanos, R., Ochoa, Z., y Oliveros, L. J. (2015). Variabilidad y cambio climáticos: su repercusión en la salud. Medisan, 19(7), 873-885. https://doi.org/ISSN 1029-3019

Rodríguez Cruz, I., y Bezunartea Valencia, M. J. (2016). Capacidad movilizadora de la información sobre medio ambiente: La importancia de la calidad periodística para promover una actitud proambiental. Estudios Sobre El Mensaje Periodistico, 22(1), 517-530. https://doi.org/10.5209/rev_ ESMP.2016.v22.n1.52611

Rodríguez, F., Jiménez, M., y Pedraza, L. (2019). Efectos del cambio climático en la salud de la población colombiana. Duazary, 16(2), 319-331. https:// doi.org/10.21676/2389783X.3186

Rodriguez, S., Cabrera, L., y Calero, E. (2018). La comunicación social en salud para la prevención de enfermedades en la comunidad. Humanidades Mèdicas, 18(2), 384-404. https://doi.org/ISSN 1727-8120

Sánchez Zavaleta, C. A. (2016). Evolución del concepto de cambio climático y su impacto en la salud pública del Perú. Revista Peruana de Medicina Experimental y Salud Publica, 33(1), 128-138. https://doi.org/10.17843/rpmesp.2016.331.2014

Suárez Zozaya, M. H. (2017). Juventud de los estudiantes universitarios. Revista de La Educacion Superior, 46(184), 39-54. https://doi.org/10.1016/j. resu.2017.12.001

Toulkeridis, T., Tamayo, E., Simón-Baile, D., Merizalde-Mora, M. J., Reyes -Yunga, D. F., Viera-Torres, M., y Heredia, M. (2020). Cambio Climático según los académicos ecuatorianos - Percepciones versus hechos. Granja, 31(1), 21-49. https://doi.org/10.17163/lgr.n31.2020.02

Zamora Martínez, M. C. (2015). Cambio climático. Revista mexicana de ciencias forestales. Revista Mexicana de Ciencias Forestales, 6(31), 1-4. $\quad$ http://www.scielo.org.mx/scielo.php?script=sci_arttext\&pi$\mathrm{d}=\mathrm{S} 2007-11322015000500001$

\section{$44 \quad$ Cumbres}

